\title{
Fresh versus frozen embryo transfer in women with thin endometrium: a retrospective cohort study
}

\author{
Zizhen Guo ${ }^{1,2}$, Ran $\mathrm{Chu}^{3}$, Liping Zhang ${ }^{1,2}$, Qian $\mathrm{Yu}^{4}$, Lei Yan ${ }^{1,2,4}$, Jinlong $\mathrm{Ma}^{4}$ \\ ${ }^{1}$ Cheeloo College of Medicine, Shandong University, Jinan, China; ${ }^{2}$ Center for Reproductive Medicine, Cheeloo College of Medicine, Shandong \\ University, Jinan, China; ${ }^{3}$ Department of Obstetrics and Gynecology, Qilu Hospital of Shandong University, Jinan, China; ${ }^{4}$ Department of \\ Pathology, Reproductive Hospital Affiliated to Shandong University, Jinan, China \\ Contributions: (I) Conception and design: J Ma; (II) Administrative support: None; (III) Provision of study materials or patients: J Ma, L Yan; (IV) \\ Collection and assembly of data: Z Guo, R Chu, Q Yu; (V) Data analysis and interpretation: Z Guo, L Zhang; (VI) Manuscript writing: All authors; \\ (VII) Final approval of manuscript: All authors. \\ Correspondence to: Jinlong Ma. Department of Pathology, Reproductive Hospital Affiliated to Shandong University, Jinan 250001, China. \\ Email: majinlong_sdu@163.com.
}

Background: A thin endometrium affects embryo implantation. We designed a retrospective cohort study to analyze the differences of in vitro fertilization-intracytoplasmic sperm injection (IVF-ICSI) outcomes between fresh embryo transfer (ET) and frozen ET in patients with a thin endometrium.

Methods: The present study comprised of 1,110 patients with a thin endometrium undergoing IVFICSI between January 2013 and December 2017 in our unit. Propensity score matching (PSM) was used to balance the influence of intergroup confounding factors and to compare the pregnancy outcomes of the matched patients in the two groups. The fresh embryo group and frozen embryo group consisted of 632 and 478 women, respectively. After PSM, 173 patients were included in the two groups, respectively.

Results: The frozen embryo group showed a higher live birth rate $(31 \%$ vs. $18.4 \%, \mathrm{P}<0.001 ; 30.6 \%$ vs. $19.7 \%, \mathrm{P}=0.019)$, clinical pregnancy rate $(40 \%$ vs. $26.4 \%, \mathrm{P}<0.001 ; 38.7 \%$ vs. $25.4 \%, \mathrm{P}=0.008)$, and biochemical pregnancy rate $(46.2 \%$ vs. $32.9 \%, \mathrm{P}<0.001 ; 44.5 \%$ vs. $31.8 \%, \mathrm{P}=0.020)$ than the fresh embryo group before and after PSM.

Conclusions: Our results demonstrated that for women with a thin endometrium who were undergoing IVF, the live birth rate, clinical pregnancy rate, and biochemical pregnancy rate after frozen ET were significantly higher than in the fresh ET group.

Keywords: Frozen embryo; fresh embryo; endometrium; in vitro fertilization-intracytoplasmic sperm injection (IVF-ICSI); propensity score matching (PSM)

Submitted Apr 09, 2020. Accepted for publication Aug 27, 2020.

doi: $10.21037 / \mathrm{atm}-20-3230$

View this article at: http://dx.doi.org/10.21037/atm-20-3230

\section{Introduction}

Embryo implantation depends on embryo quality, endometrial receptivity, and the interaction between the embryo and maternal endometrium. Ideally, the method of assessment for endometrial receptivity should be easily performed in daily clinical routine and preferably be noninvasive (1). Endometrial thickness (EMT) can be easily measured by transvaginal ultrasonography (TVU). Several studies suggest that an EMT $<8 \mathrm{~mm}$ is associated with implantation failure in both fresh and frozen embryo transfer (ET) cycles (2-6). The periodical changes of the endometrium, especially in the hyperplasia period, are mainly affected by estrogen, which plays a vital role in the formation of thin endometrium. Therefore, studies have suggested using hormone replacement treatment to increase the thickness of a thin endometrium in frozen-thaw ET cycles (7). At present, vitrified cryopreservation has been 
proved to be a cryopreservation method with high recovery efficiency and less damage to embryos.

Moreover, in the frozen-thaw ET cycle, large doses of exogenous gonadotropin application are avoided, and the endometrium is closer to the state of natural pregnancy with better endometrial receptivity. At present, the study of thin endometria is in the research and exploration stages, and some therapeutic effects have yet to be proven. Clinicians may often be faced with the dilemma of whether they should continue the fresh in vitro fertilization (IVF) cycle or cryopreserve the embryos to wait for a better chance of pregnancy during the subsequent frozen ET cycle. Although the live birth rate of a frozen single-blastocyst transfer is higher than that of a fresh single-blastocyst transfer in ovulating women, according to randomized, controlled trial (8), there have been no analyses on patients with a thin endometrium. The present study aimed to analyze the differences in pregnancy outcomes between fresh and frozen-thaw ET for patients with a thin endometrium. We present the following article in accordance with the STROBE reporting checklist (available at http://dx.doi. org/10.21037/atm-20-3230).

\section{Methods}

\section{Study participants and design}

The cut-off value for the thin endometrium was an EMT $\leq 7 \mathrm{~mm}$ on human chorionic gonadotropin (hCG) trigger day, as per standard procedure (9). Initially, we screened all thin endometrial patient (EMT $\leq 7 \mathrm{~mm}$ on hCG trigger day) who underwent IVF-intracytoplasmic sperm injection (ICSI) ET treatment at the Reproductive Hospital Affiliated with Shandong University between January 2013 and December 2017. Patients who underwent frozen ET had a freeze-all strategy after oocyte retrieval, and we included their first ET cycles. Our exclusion criteria included: (I) oocyte donation cycles; (II) preimplantation genetic testing cycles; and (III) patients with uterine malformations, untreated submucosal uterine fibroids, or endometrial polyps. In total, we identified 1,110 women who met the study criteria, including 632 women who had a fresh ET (fresh embryo group) and 478 women who had a frozen ET (frozen embryo group). The study was conducted following the Declaration of Helsinki (as revised in 2013). The institutional review board approved this retrospective research of the Center for Reproductive Hospital Affiliated to Shandong University on October 26, 2017 (reference no. 2017-36). Informed consent was waived because of the retrospective design of the study.

\section{IVF}

Controlled ovarian stimulation, oocyte retrieval, embryo culture, endometrial preparation, and ET were based on our standard protocols. All patients were given a conventional ovarian stimulation regimen, and TVU was used to monitor the growth and development of follicles. Different ovarian stimulation protocols were selected according to the patient's age and ovarian function, including gonadotrophin-releasing hormone (GnRH) agonist long protocols, GnRH agonist short protocols, flexible GnRH antagonist protocols, mild stimulation protocols, and natural cycle protocols, which have been previously reported (10). Next, hCG was given intramuscularly at a dose of 4,000-10,000 IU when two or more follicles measured $\geq 18 \mathrm{~mm}$. Oocyte retrieval was carried out 34-36 hours later. Conventional IVF or ICSI was performed according to semen quality. For women in the fresh embryo group, luteal-phase support was started after oocyte retrieval and was continued until 10 weeks of gestation. For women in the frozen embryo group, all available embryos were vitrified. Some good-quality embryos were cryopreserved using vitrification on day 2 or day 3 , and the other embryos could be vitrified at the cleavage or blastocyst stage. Vitrification was carried out following the Mukaida protocol with cryoloop (11). The embryos were first placed into the basic medium (HEPESbuffered modified hTF medium), and then transferred into the basic medium containing $7.5 \%$ (v/v) dimethylsulfoxide (DMSO) and 7.5\% (v/v) ethylene glycol (EG; vitrification solution I) for 2 minutes. Finally, the embryos were suspended for 30 seconds in the basic medium containing $15 \%$ (v/v) DMSO, 15\% (v/v) EG, $10 \mathrm{mg} / \mathrm{mL}$ Ficoll 70 , and $0.65 \mathrm{~mol} / \mathrm{L}$ sucrose (vitrification solution II), and were plunged into liquid nitrogen quickly. Warming was carried out in a four-well multidish according to the Mukaida protocol. The embryos were incubated in a basic medium containing $0.33 \mathrm{~mol} / \mathrm{L}$ sucrose (thawing solution I), a basic medium containing $0.2 \mathrm{~mol} / \mathrm{L}$ sucrose (thawing solution II), and a basic medium for 2,3 , and 5 minutes at $37^{\circ} \mathrm{C}$, respectively. The endometrium was prepared by a natural cycle, programmed cycle, and minimal ovarian stimulation cycle, followed by frozen embryo transplantation. Patients with normal menstruation and ovulation chose the natural cycle, and patients with irregular menstruation or long- 
term persistent anovulatory chose the programmed cycle or minimal ovarian stimulation cycle.

\section{EMT measurement}

In our study, a thin endometrium was diagnosed as EMT $\leq 7 \mathrm{~mm}$ on hCG trigger day. EMT was defined as the maximal distance from one endometrial-myometrial interface to the other in the midsagittal plane of the uterus, as measured by ultrasonography. All patients were measured by the same operator group to avoid errors. Each patient was measured three times, and the average value was taken. The EMT was taken from the day of the hCG trigger in fresh cycles. Meanwhile, in the frozen cycles, the EMT was measured at the last ultrasound before the use of progesterone.

\section{Pregnancy assessment}

Biochemical pregnancy was defined as an hCG serum level of $>10 \mathrm{mIU} / \mathrm{mL}$. Clinical pregnancy was defined as the observation of a sac by ultrasound. Live birth was defined as delivery of any viable infant at $\geq 28$ weeks or more of gestation. We calculated these parameters based on our previous study (12).

\section{Statistical analysis}

All analyses were performed with Statistical Package for the Social Sciences (SPSS) version 24.0. Categorical data were represented as frequency and percentage; variables in these measures between the study groups were compared utilizing a chi-squared test. Continuous data were expressed as means ( \pm standard deviation), with a $t$-test for betweengroup differences. A $\mathrm{P}$ value $<0.05$ was considered to be statistically significant.

The present study was retrospective, and patients were not randomly assigned to groups. Therefore, there were inevitable potential confounding and selection biases that could affect the outcomes. Therefore, we used propensity score matching (PSM) to identify fresh embryo group patients who were almost identical to the frozen embryo group patients. We assigned propensity scores to the baseline characteristics and ET variables that potentially affect pregnancy outcomes. We used a logistic regression model to calculate propensity scores based on female age, body mass index (BMI), duration of infertility, antral follicle count (AFC) in both ovaries, number of oocytes retrieved, basal follicle stimulating hormone (FSH), basal luteinizing hormone (LH), type of infertility, the proportion of patients with polycystic ovary syndrome, EMT on the hCG day, number of embryos transferred, and stage of embryo transferred. These variables were listed as baseline characteristics and ET variables. Twelve independent variables were used to determine the propensity score to remove bias. A 1:1 match between the fresh embryo group and the frozen embryo group was subsequently obtained using nearest neighbor matching, with a caliper width of 0.02 and without replacement. The PSM process was implemented using the SPSS PSM extension.

\section{Results}

A total of 1,110 women were included in the study. The fresh embryo group and frozen embryo group consisted of 632 and 478 women, respectively. After the PSM procedure, 173 patients were included in the fresh embryo group and frozen embryo group, respectively. The baseline characteristics, ET variables, and pregnancy outcomes before and after PSM were evaluated.

\section{Patient baseline characteristics and ET variables}

Before matching, baseline characteristics and ET variables were unevenly distributed between the two groups of patients. The patients in the fresh embryo group were older $(34.75 \pm 4.65$ vs. $33.57 \pm 5.42$ years, $\mathrm{P}<0.001)$ and had higher basal FSH $(7.95 \pm 3.39$ vs. $7.32 \pm 3.59, \mathrm{P}=0.003)$. The fresh embryo group patients had fewer AFC in both ovaries $(11.29 \pm 8.04$ vs. $13.92 \pm 10.00, \mathrm{P}<0.001)$, fewer retrieved oocytes (7.19 \pm 4.82 vs. $10.49 \pm 7.76, \mathrm{P}<0.001)$, fewer blastocyst embryos $(17.2 \%$ vs. $85.4 \%, \mathrm{P}<0.001)$, fewer single embryos transferred $(35.3 \%$ vs. $76.6 \%, \mathrm{P}<0.001)$ less PCOS (9.5\% vs. $15.3 \%, \mathrm{P}=0.003)$, and thicker EMT on hCG day $(0.66 \pm 0.06$ vs. $0.62 \pm 0.10 \mathrm{~cm}, \mathrm{P}<0.001)$ than the frozen embryo group patients. After matching, the baseline characteristics and ET variables of the patients were similar in the two groups, as shown in Table 1. No significant differences were observed in age, infertility duration, BMI, AFC in both ovaries, number of oocytes retrieved, baseline $\mathrm{FSH}$, baseline $\mathrm{LH}$, the proportion of patients with PCOS, EMT on hCG trigger day, stage of embryo transferred, or number of embryos transferred between the two groups $(P>0.05)$ (Table 1). 
Table 1 Baseline characteristics and embryo transfer variables of study patients before and after PSM

\begin{tabular}{|c|c|c|c|c|c|c|}
\hline Characteristics & \multicolumn{3}{|c|}{ Before PSM $(n=1,110)$} & \multicolumn{3}{|c|}{ After PSM (n=346) } \\
\hline Age, years & $34.75 \pm 5.54$ & $33.57 \pm 5.42$ & $<0.001^{* \star *}$ & $34.10 \pm 5.41$ & $34.11 \pm 5.89$ & 0.992 \\
\hline BMI, $\mathrm{kg} / \mathrm{m}^{2}$ & $23.76 \pm 3.60$ & $23.34 \pm 3.47$ & 0.053 & $23.81 \pm 3.67$ & $23.18 \pm 3.45$ & 0.100 \\
\hline Previous conception, n (\%) & $456(72.2)$ & $333(69.7)$ & 0.385 & $124(71.7)$ & $135(78.0)$ & 0.173 \\
\hline AFC, no. & $11.29 \pm 8.04$ & $13.92 \pm 10.00$ & $<0.001^{\star \star *}$ & $12.23 \pm 8.14$ & $12.54 \pm 7.94$ & 0.713 \\
\hline Basal FSH, IU/L & $7.95 \pm 3.39$ & $7.32 \pm 3.59$ & $0.003^{* *}$ & $7.49 \pm 2.46$ & $7.63 \pm 4.02$ & 0.712 \\
\hline Basal LH, IU/L & $5.36 \pm 3.54$ & $5.81 \pm 4.49$ & 0.063 & $5.37 \pm 3.40$ & $5.80 \pm 4.41$ & 0.312 \\
\hline $\begin{array}{l}\text { Stage of embryo transferred, } \\
\mathrm{n}(\%)\end{array}$ & & & $<0.001^{\star \star *}$ & & & 1 \\
\hline Cleavage-stage embryo & $523(82.8)$ & $70(14.6)$ & & $65(37.6)$ & $65(37.6)$ & \\
\hline Blastocyst & $109(17.2)$ & $408(85.4)$ & & $108(62.4)$ & $108(62.4)$ & \\
\hline $\begin{array}{l}\text { Number of embryos transferred, } \\
\mathrm{n}(\%)\end{array}$ & & & $<0.001^{\star \star \star}$ & & & 0.666 \\
\hline One embryo transferred & $223(35.3)$ & 366 (76.6) & & $118(68.2)$ & $117(67.6)$ & \\
\hline Two embryos transferred & $404(63.9)$ & $110(23.0)$ & & $52(30.1)$ & $55(31.8)$ & \\
\hline
\end{tabular}

Values are presented as mean \pm standard deviation or $\mathrm{n}(\%) .{ }^{*}, \mathrm{P}<0.01 ;{ }^{* \star}, \mathrm{P}<0.001 . \mathrm{P}>0.05$, not significant. PSM, propensity score matching; BMI, body mass index; AFC, antral follicle count in both ovaries; FSH, follicle stimulating hormone; LH, luteinizing hormone; PCOS, polycystic ovary syndrome, diagnosed by modified Rotterdam criteria; EMT, endometrial thickness.

\section{EMT in frozen embryo group patients}

In the frozen embryo group, the EMT thickened significantly before ET day compared with the EMT on the hCG trigger day, from $0.61 \pm 0.10$ to $0.80 \pm 0.15 \mathrm{~mm}$ in the women who underwent natural cycles $(\mathrm{P}<0.001)$, from $0.62 \pm 0.09$ to $0.75 \pm 0.13 \mathrm{~mm}$ in the women underwent programmed cycles $(\mathrm{P}<0.001)$, and from $0.61 \pm 0.09$ to $0.72 \pm 0.12 \mathrm{~mm}$ in the women who underwent minimal ovarian stimulation cycles $(\mathrm{P}<0.001)$, as shown in Table 2 .

\section{Pregnancy outcomes of patients in the fresh embryo group and frozen embryo group}

We compared the pregnancy outcomes of patients in the fresh embryo group and frozen embryo group. The frozen embryo group showed a higher live birth rate $(31 \%$ vs. $18.4 \%, \mathrm{P}<0.001 ; 30.6 \%$ vs. $19.7 \%, \mathrm{P}=0.019)$, clinical pregnancy rate $(40 \%$ vs. $26.4 \%, \mathrm{P}<0.001 ; 38.7 \%$ vs. $25.4 \%$, $\mathrm{P}=0.008)$, and biochemical pregnancy rate $(46.2 \%$ vs. $32.9 \%, \mathrm{P}<0.001 ; 44.5 \%$ vs. $31.8 \%, \mathrm{P}=0.020)$ than the fresh embryo group before and after PSM, as shown in Table 3.

\section{Discussion}

With the continuous improvement of assisted reproductive technology and laboratory embryo cultivation technology, the quality of embryos is continuously improving, so endometrial function and endometrial receptivity have become an essential factor affecting embryo implantation (1). A sufficient EMT is essential for a 
Table 2 Endometrial thickness in the patients of Frozen-Embryo group

\begin{tabular}{lccc}
\hline Endometrial thickness, cm & On HCG trigger day & Before embryo transfer day & P value \\
\hline Natural cycles $(n=181)$ & $0.61 \pm 0.10$ & $0.80 \pm 0.15$ & $<0.001^{\star \star \star}$ \\
Programmed cycles $(n=181)$ & $0.62 \pm 0.09$ & $0.75 \pm 0.13$ & $<0.001^{\star \star *}$ \\
Minimal ovarian stimulation $(n=116)$ & $0.61 \pm 0.09$ & $0.72 \pm 0.12$ & $<0.001^{\star \star \star}$ \\
\hline
\end{tabular}

Values are presented as mean \pm standard deviation or $\mathrm{n}(\%) .{ }^{* \star}, \mathrm{P}<0.001$.

Table 3 The pregnancy outcomes of the patients in two groups after PSM

\begin{tabular}{|c|c|c|c|c|c|c|}
\hline \multirow[b]{2}{*}{ Outcomes } & \multicolumn{3}{|c|}{ Before PSM $(n=1,110)$} & \multicolumn{3}{|c|}{ After PSM $(n=346)$} \\
\hline & $\begin{array}{l}\text { Fresh-Embryo } \\
\text { group }(\mathrm{n}=632)\end{array}$ & $\begin{array}{c}\text { Frozen-Embryo } \\
\text { group }(n=478)\end{array}$ & $P$ value & $\begin{array}{l}\text { Fresh-Embryo } \\
\text { group }(n=173)\end{array}$ & $\begin{array}{c}\text { Frozen-Embryo } \\
\text { group }(n=173)\end{array}$ & $P$ value \\
\hline Clinical pregnancy rate, $\mathrm{n}(\%)$ & $167(26.4)$ & $191(40.0)$ & $<0.001^{\star \star \star}$ & $44(25.4)$ & $67(38.7)$ & $0.008^{\star *}$ \\
\hline Biochemical pregnancy rate, $\mathrm{n}(\%)$ & $208(32.9)$ & 221 (46.2) & $<0.001^{\star \star \star}$ & $55(31.8)$ & $77(44.5)$ & $0.020^{*}$ \\
\hline
\end{tabular}

successful pregnancy. At present, there is no consensus on the definition of thin endometrium (13). The optimal EMT is unclear. However, several studies suggest that pregnancy is less probable when the endometrium is $<8 \mathrm{~mm}(2-6)$. In the meta-analysis by Kasius et al., the clinical pregnancy rates of women with $\mathrm{EMT} \leq 7 \mathrm{~mm}$ were significantly lower than those with EMT $>7 \mathrm{~mm}$, and the live birth rate of women with EMT $\leq 7 \mathrm{~mm}$ showed a declining trend (9). A recent systematic review showed that both clinical pregnancy and live birth rate decreased significantly for each millimeter of EMT $<8 \mathrm{~mm}$ in fresh IVF cycles and $<7 \mathrm{~mm}$ in frozen-thaw IVF cycles (14). In our reproductive center, we used a cut-off of $7 \mathrm{~mm}$ to diagnose a thin endometrium. EMT provides an effective method for predicting endometrial receptivity and subsequent IVF outcome (15). It is unclear why a thin endometrium is associated with a poor pregnancy outcome. It is speculated that the oxygen concentrations increase in the basal layer in patients with a thin endometrium. High oxygen concentrations may be harmful to embryo implantation (16). In patients with a poor endometrial response, exogenous estradiol treatment is a common practice for improving EMT. Jimenez et al. reported that previous fresh-cycle EMT is associated with subsequent frozen-cycle EMT, suggesting that women with a fresh-cycle thickness of $\leq 11.5 \mathrm{~mm}$ might need additional estrogen supplementation to achieve adequate EMT for frozen ET preparation (17). Our study showed that the
EMT increased with all three endometrial preparation regimens during frozen ET cycles. This could be because frozen ET can restore the stimulated ovaries and give patients with a thin endometrium a chance to regrow their lining.

There are two types of ET strategies in in vitro fertilization-intracytoplasmic sperm injection (IVF-ICSI): (I) a conventional IVF-ICSI strategy; that is, fresh ET and one or more frozen-thaw ET; and (II) a freeze-all strategy; that is, all embryos are cryopreserved after IVF, and frozenthaw ET is conducted later, instead of a fresh ET (18). In general, indications for the freeze-all strategy include moderate-to-severe ovarian hyperstimulation syndrome (OHSS), a high risk of moderate-to-severe OHSS, a thin endometrium, the presence of progesterone elevation, and other factors affecting embryo implantation. Specifically, in our study, some patients participated in the hospital's randomized, controlled trial and were randomly assigned to the frozen embryo group $(12,19)$. In the past few decades, many studies have compared pregnancy outcomes between fresh and frozen ET. However, the conclusions about these two methods are still controversial. Most randomized trials have compared fresh and frozen ET in women with an expected high response to ovarian stimulation $(12,20,21)$. Chen et al. reported that, among women with PCOS undergoing IVF, frozen ET has been shown to result in a higher rate of live births than fresh ET (12), although there 
is no significant difference in ovulatory women (19). In a recently published study, it was shown that the singleton live birth rate of a frozen single-blastocyst transfer was higher than that of a fresh single-blastocyst transfer in ovulatory women (8). In a retrospective study involving poor ovarian responders, it was suggested that there was no significant difference in pregnancy outcomes between the fresh and frozen ET groups when letrozole was used to stimulate ovulation (22). Roque analyzed the adverse effects of controlled ovarian stimulation on endometrium receptivity, IVF safety, and obstetric and perinatal morbidity by reviewing the existing literature, and believed that the whole-embryo freeze-all strategy was better than fresh ET (18). These results suggest that the principle of individualization should be followed in the selection of ET strategies for different patients.

In the present study, we found that patients with a thin endometrial in the frozen embryo group had better pregnancy outcomes than those in the fresh embryo group. The higher pregnancy rate after frozen ET may involve three factors: high-quality embryo, a more receptive endometrium, and the synchronicity of development between them (23). During ovarian stimulation, large doses of exogenous gonadotropin application lead to the early expression of the progesterone receptor and the early transformation of the endometrium, which has adverse effects on the development of the endometrium, thereby reducing the embryo implantation rate $(24,25)$. During the frozen-thaw ET cycles, the endometrium exposed to ovarian stimulation is subsequently shed and regenerated under the endometrial preparation scheme, which can provide a more favorable uterine environment for embryo implantation; this method is similar to the natural implantation process. Also, endometrial thickening may contribute to improved pregnancy outcomes. In the present study, we considered the difference in pregnancy outcomes between fresh and frozen ET for patients with a thin endometrium. The results of our study suggest that, for women with a thin endometrium, the EMT increases in frozen ET cycles compared with their EMT on hCG trigger day and frozen ET results in higher rates of live births and clinical and biochemical pregnancies than a fresh ET.

Many studies have compared the advantages and disadvantages of fresh and frozen ET; however, in our study, we focused on patients with a thin endometrium. The greatest advantage of the present study was that it considered PSM, which is an effective alternative to randomized, controlled trials in retrospective studies, and carries out strict matching between fresh and frozen ET groups. There was no statistical difference in the basic characteristics between the two groups after matching, which reduced the possibility of interference by these confounding factors and enhanced the credibility of the results in the subsequent comparison of pregnancy outcomes between the two groups (26). However, the present study does have some limitations. First, it was a single-center retrospective study, and the conclusion needs to be further tested in a multicenter randomized, controlled study. Second, patients with a thin endometrial account for a small number of patients, thus the sample size of the present study is limited. This hypothesis needs to be further confirmed by increasing the sample size in future studies.

In conclusion, for women with a thin endometrium undergoing IVF, the live birth rate, clinical pregnancy rate, and biochemical pregnancy rate after frozen ET were significantly higher than in the fresh embryo group. The EMT in patients with a thin endometrium increased in the frozen ET cycles compared with their EMT on hCG trigger day. We recommend that patients with a thin endometrium adopt a freeze-all strategy for selective frozen ET.

\section{Acknowledgments}

The authors thank R. Scott and J. Chapnick, PhD, from AME Editing Service (http://editing.amegroups. $\mathrm{cn} /$ \# editing) for help in revising the manuscript, and all of our patients for their participation in the study.

Funding: The present study was supported by the National Key Research and Development of China (no. 2018YFC1004800), the National Natural Science Foundation of China (no. 81571414), and a grant from the Shandong Province key research and development project (no. 2016GSF201086).

\section{Footnote}

Reporting Checklist: The authors completed the STROBE reporting checklist. Available at http://dx.doi.org/10.21037/ atm-20-3230

Data Sharing Statement: Available at http://dx.doi. org/10.21037/atm-20-3230

Peer Review File: Available at http://dx.doi.org/10.21037/ atm-20-3230 
Conflicts of Interest: All authors have completed the ICMJE uniform disclosure form (available at http://dx.doi. org/10.21037/atm-20-3230). The authors have no conflicts of interest to declare.

Ethical Statement: The authors are accountable for all aspects of the work in ensuring that questions related to the accuracy or integrity of any part of the work are appropriately investigated and resolved. The study was conducted following the Declaration of Helsinki (as revised in 2013). The institutional review board approved this retrospective research of the Center for Reproductive Hospital Affiliated to Shandong University on October 26, 2017 (reference no. 2017-36). Informed consent was waived because of the retrospective design of the study.

Open Access Statement: This is an Open Access article distributed in accordance with the Creative Commons Attribution-NonCommercial-NoDerivs 4.0 International License (CC BY-NC-ND 4.0), which permits the noncommercial replication and distribution of the article with the strict proviso that no changes or edits are made and the original work is properly cited (including links to both the formal publication through the relevant DOI and the license). See: https://creativecommons.org/licenses/by-nc-nd/4.0/.

\section{References}

1. Strowitzki T, Germeyer A, Popovici R, et al. The human endometrium as a fertility-determining factor. Hum Reprod Update 2006;12:617-30.

2. Abdalla HI, Brooks AA, Johnson MR, et al. Endometrial thickness: a predictor of implantation in ovum recipients? Hum Reprod 1994;9:363-5.

3. Basir GS, O WS, So WW, et al. Evaluation of cycleto-cycle variation of endometrial responsiveness using transvaginal sonography in women undergoing assisted reproduction. Ultrasound Obstet Gynecol 2002;19:484-9.

4. Dessolle L, Daraï E, Cornet D, et al. Determinants of pregnancy rate in the donor oocyte model: a multivariate analysis of 450 frozen-thawed embryo transfers. Hum Reprod 2009;24:3082-9.

5. El-Toukhy T, Coomarasamy A, Khairy M, et al. The relationship between endometrial thickness and outcome of medicated frozen embryo replacement cycles. Fertil Steril 2008;89:832-9.

6. Gonen Y, Casper RF, Jacobson W, et al. Endometrial thickness and growth during ovarian stimulation: a possible predictor of implantation in in vitro fertilization. Fertil Steril 1989;52:446-50.

7. Chen MJ, Yang JH, Peng FH, et al. Extended estrogen administration for women with thin endometrium in frozen-thawed in-vitro fertilization programs. J Assist Reprod Genet 2006;23:337-42.

8. Wei D, Liu JY, Sun Y, et al. Frozen versus fresh single blastocyst transfer in ovulatory women: a multicentre, randomised controlled trial. Lancet 2019;393:1310-8.

9. Kasius A, Smit JG, Torrance HL, et al. Endometrial thickness and pregnancy rates after IVF: a systematic review and meta-analysis. Hum Reprod Update 2014;20:530-41.

10. Guo Z, Xu X, Zhang L, et al. Endometrial thickness is associated with incidence of small-for-gestational-age infants in fresh in vitro fertilization-intracytoplasmic sperm injection and embryo transfer cycles. Fertil Steril 2020;113:745-52.

11. Takahashi K, Mukaida T, Goto T, et al. Perinatal outcome of blastocyst transfer with vitrification using cryoloop: a 4-year follow-up study. Fertil Steril 2005;84:88-92.

12. Chen ZJ, Shi Y, Sun Y, et al. Fresh versus Frozen Embryos for Infertility in the Polycystic Ovary Syndrome. N Engl J Med 2016;375:523-33.

13. Senturk LM, Erel CT. Thin endometrium in assisted reproductive technology. Curr Opin Obstet Gynecol 2008;20:221-8.

14. Liu KE, Hartman M, Hartman A, et al. The impact of a thin endometrial lining on fresh and frozen-thaw IVF outcomes: an analysis of over 40000 embryo transfers. Hum Reprod 2018;33:1883-8.

15. Schild RL, Knobloch C, Dorn C, et al. Endometrial receptivity in an in vitro fertilization program as assessed by spiral artery blood flow, endometrial thickness, endometrial volume, and uterine artery blood flow. Fertil Steril 2001;75:361-6.

16. Casper RF. It's time to pay attention to the endometrium. Fertil Steril 2011;96:519-21.

17. Jimenez PT, Schon SB, Odem RR, et al. A retrospective cross-sectional study: fresh cycle endometrial thickness is a sensitive predictor of inadequate endometrial thickness in frozen embryo transfer cycles. Reprod Biol Endocrinol 2013;11:35.

18. Roque M. Freeze-all policy: is it time for that? J Assist Reprod Genet 2015;32:171-6.

19. Shi Y, Sun $Y$, Hao C, et al. Transfer of Fresh versus Frozen Embryos in Ovulatory Women. N Engl J Med 2018;378:126-36. 
20. Ferraretti AP, Gianaroli L, Magli C, et al. Elective cryopreservation of all pronucleate embryos in women at risk of ovarian hyperstimulation syndrome: efficiency and safety. Hum Reprod 1999;14:1457-60.

21. Shapiro BS, Daneshmand ST, Garner FC, et al. Evidence of impaired endometrial receptivity after ovarian stimulation for in vitro fertilization: a prospective randomized trial comparing fresh and frozen-thawed embryo transfers in high responders. Fertil Steril 2011;96:516-8.

22. Çelik S, Turgut NE, Ya mur E, et al. The effects of fresh embryo transfers and elective frozen/thawed embryo transfers on pregancy outcomes in poor ovarian responders as defined by the Bologna criteria. Turk J Obstet Gynecol 2015;12:132-8.

Cite this article as: Guo Z, Chu R, Zhang L, Yu Q, Yan L, $\mathrm{Ma} \mathrm{J}$. Fresh versus frozen embryo transfer in women with thin endometrium: a retrospective cohort study. Ann Transl Med 2020;8(21):1435. doi: 10.21037/atm-20-3230
23. Teh WT, McBain J, Rogers P. What is the contribution of embryo-endometrial asynchrony to implantation failure? J Assist Reprod Genet 2016;33:1419-30.

24. Liu $\mathrm{Y}$, Lee $\mathrm{KF}, \mathrm{Ng} \mathrm{EH}$, et al. Gene expression profiling of human peri-implantation endometria between natural and stimulated cycles. Fertil Steril 2008;90:2152-64.

25. Mirkin S, Nikas G, Hsiu JG, et al. Gene expression profiles and structural/functional features of the peri-implantation endometrium in natural and gonadotropin-stimulated cycles. J Clin Endocrinol Metab 2004;89:5742-52.

26. Whittaker W, Anselmi L, Kristensen SR, et al. Associations between Extending Access to Primary Care and Emergency Department Visits: A Difference-InDifferences Analysis. PLoS Med 2016;13:e1002113. 